\title{
Ultrasound-Guided Continuous Thoracic Paravertebral Infusion of Methylene Blue in the Treatment of Postherpetic Neuralgia: A Prospective, Randomized, Controlled Study
}

\author{
Mingxia Wang · Jinyuan Zhang - Li Zheng - Hongwei Fang • \\ Yiguo Zhang $\cdot$ Huimin Deng $\cdot$ Mansi Wang $\cdot$ Xiuqin Yu • \\ Qingxiang Meng · Yuanli Chen · Lijun Liao · Xin Lv • \\ Hao Yang · Xiangrui Wang \\ Received: February 19, 2021 / Accepted: April 2, 2021 / Published online: April 11, 2021 \\ (C) The Author(s) 2021
}

\section{ABSTRACT}

Introduction: Postherpetic neuralgia (PHN) is the most common complication of herpes zoster. Methylene blue (MB) is an inhibitor of nitric oxide synthesis with potentially analgesic and anti-inflammatory properties. Studies have demonstrated that thoracic paravertebral single $\mathrm{MB}$ injection is effective in treating chronic pain. However, there are rare reports of the efficacy of continuous thoracic paravertebral infusion of $\mathrm{MB}$ for pain management in PHN patients. The purpose of this study was to evaluate the therapeutic effects of continuous thoracic paravertebral infusion of MB on PHN. Methods: A total of 104 PHN patients were randomly divided into two groups: the control

Mingxia Wang, Jinyuan Zhang and Li Zheng contributed equally to this work.

M. Wang · J. Zhang · H. Fang · X. Yu - Q. Meng ·

Y. Chen · L. Liao $\cdot$ X. Wang $(\bowtie)$

Department of Anesthesiology and Critical Care Medicine, Shanghai East Hospital, Tongji University School of Medicine, 150 Jimo Rd, Shanghai 200120, China

e-mail: wangxiangruirjyy@sina.com

L. Zheng $\cdot$ Y. Zhang $\cdot$ H. Deng $\cdot$ M. Wang $\cdot$ X. Lv

H. Yang $(\square)$

Department of Anesthesiology, Shanghai

Pulmonary Hospital, Tongji University School of

Medicine, 507 Zhengmin Rd, Shanghai 200433,

China

e-mail: yanghaozunyi@sina.com group (continuous thoracic paravertebral infusion of $5 \%$ lidocaine in a total volume of $300 \mathrm{ml}$ ) and the $\mathrm{MB}$ group (continuous thoracic paravertebral infusion of $5 \%$ lidocaine plus $0.2 \% \mathrm{MB}$ in a total volume of $300 \mathrm{ml}$ ). All patients were evaluated using the Numerical Rating Scale (NRS), Insomnia Severity Index (ISI), Patient Health Questionnaire-9 (PHQ-9), 36-Item ShortForm Health Survey (SF-36), and medication doses before and after the procedure. The effective treatment rate and adverse complications were recorded 6 months after the procedure.

Results: In both groups, the NRS scores, ISI scores, PHQ-9 scores, and rescue medication dosages were significantly decreased at different time points after treatment compared to baseline, while the SF-36 scores were evidently improved at different time points after treatment compared to baseline. Compared with the control group, the MB group had significantly reduced NRS scores, ISI scores, PHQ-9 scores, and rescue medication dosages at each observation time point. Furthermore, the SF-36 scores in the $\mathrm{MB}$ group were significantly higher than those in the control group at each observation time point. The total effective treatment rate of the $\mathrm{MB}$ group was higher than that of the control group 6 months after the procedure. No severe adverse complications were observed in either group.

Conclusions: Ultrasound-guided continuous thoracic paravertebral infusion with $\mathrm{MB}$ is a safe and effective therapy for PHN. Continuous 
infusion with $\mathrm{MB}$ can significantly reduce pain intensity, improve pain-related depression, increase quality of life, and decrease the amount of rescue medicine with no serious adverse complications.

Keywords: Postherpetic neuralgia; Methylene blue; Ultrasound guidance; Thoracic paravertebral infusion; Pain

\section{Key Summary Points}

\section{Why carry out this study?}

Postherpetic neuralgia is the most frequent chronic complication of herpes zoster, and is manifested by neuropathic pain after the rash has healed. It is estimated that approximately $20 \%$ of herpes zoster patients develop postherpetic neuralgia.

Ultrasound-guided thoracic paravertebral single methylene blue injection can significantly reduce pain intensity in chronic pain patients.

We hypothesized that ultrasound-guided continuous methylene blue thoracic paravertebral infusion can provide safe, effective, and long-term analgesia for postherpetic neuralgia patients.

\section{What was learned from the study?}

Thoracic paravertebral infusion of methylene blue exerts a significant analgesic effect and reduces rescue medication requirements in postherpetic neuralgia patients. Thoracic paravertebral infusion of methylene blue improves quality of life in postherpetic neuralgia patients.

Use of an ultrasound-guided paravertebral catheter decreased the risk of adverse complications.

Ultrasound-guided continuous thoracic paravertebral infusion with methylene blue is a safe and effective therapy for postherpetic neuralgia.

\section{DIGITAL FEATURES}

This article is published with digital features, including a summary slide, to facilitate understanding of the article. To view digital features for this article go to https://doi.org/10.6084/ m9.figshare.14356679.

\section{INTRODUCTION}

Postherpetic neuralgia (PHN) is the most frequent chronic complication of herpes zoster (HZ), and is manifested by neuropathic pain after the rash has healed [1]. Recent studies have shown that the annual incidence of $\mathrm{HZ}$ is approximately 3.4 cases per 1000 persons, and it rises sharply from the age of 50 years to approximately 10.9 cases per 1000 person in the $\geq 80$ age group $[2,3]$. It is estimated that approximately $20 \%$ of $\mathrm{HZ}$ patients develop PHN [4]. In China, a higher proportion $(29.8 \%)$ of patients develop PHN, and the prevalence increases with age [5]. PHN can last from several months to several years, even up to 10 years. PHN substantially affects patient quality of life and can cause physical disability, emotional distress, and social isolation; it also presents a serious economic burden and public health problems for society $[6,7]$. Therefore, a therapy that can effectively alleviate PHN is urgently needed.

The goal of PHN treatment is to improve quality of life by relieving pain. Currently, the main strategies for PHN management are medication and invasive interventional therapies. Pharmacological agents include opioids, antiviral drugs, nonsteroidal anti-inflammatory drugs, antiepileptic drugs, anticonvulsants, tricyclic antidepressants, and invasive interventional therapies, including pulsed radiofrequency of the dorsal root ganglion, electrical stimulation of the spinal cord, morphine pump implantation, peripheral neurotomy, autologous fat grafting, and acupuncture therapy [8-10]. However, these approaches have many adverse effects and risks, such as respiratory depression, nausea, vomiting, addiction, allergy, bleeding, infection, pneumothorax, and 
spinal cord injury. Therefore, it is important to find another effective and safe treatment for PHN.

Recently, numerous studies have reported favorable outcomes with early nerve blocks and confirmed a positive impact on the prevention and treatment of PHN [11-13]. After the advent of ultrasound imaging in nerve blocks, ultrasound-guided thoracic paravertebral block (USG-TPVB) has become increasingly popular for perioperative pain management and neuropathic pain treatment. The ultrasound-guided approach is a preferred method due to easy visualization and accurate puncture of the paravertebral space. USG-TPVB can effectively mitigate acute postoperative pain and chronic neuropathic pain, and the incidence of undesirable complications of this approach, such as nausea, vomiting, extradural hematoma, pulmonary complications, and nerve injury, is low [14-17].

Methylene blue (MB) is a guanylyl cyclase inhibitor that can suppress the production of superoxide radicals. Several studies have shown that $\mathrm{MB}$ can be used as an antioxidative and anti-inflammatory agent for the treatment of malaria, psychotic disorders, osteoarthritis-associated pain, methemoglobinemia, cardiopulmonary bypass, shock, neurodegenerative diseases, and traumatic brain injury [18-22]. Recently, it was reported that ultrasound-guided thoracic paravertebral single methylene blue injection can significantly reduce pain intensity and plasma proinflammatory cytokine levels in PHN patients, and can improve their basic living ability and self-evaluation without incurring obvious adverse side effects [23].

The abovementioned research indicated that ultrasound-guided $\mathrm{MB}$ thoracic paravertebral injection is one possible choice for the treatment of PHN. However, whether continuous MB thoracic paravertebral infusion can provide safe, effective, and long-term analgesia for PHN patients is still unclear. Therefore, this prospective randomized study was conducted to evaluate the efficacy and safety of ultrasoundguided continuous $\mathrm{MB}$ thoracic paravertebral infusion in PHN patients.

\section{METHODS}

\section{Ethics and Patients}

This study was approved by the Institutional Review Board and Ethics Committee of Shanghai East Hospital (2019, No. 006). The present clinical research was conducted in accordance with the Declaration of Helsinki. Written informed consent was obtained from all patients before inclusion. A total of 106 patients with PHN were enrolled from January 2019 to January 2020 at the pain clinic of Shanghai East Hospital. The PHN diagnosis was based on the diagnostic criteria of the International Association for the Study of Pain (IASP). The inclusion criteria were as follows: (1) age $>18$ years, (2) course of chronic pain $>30$ days, (3) pain distributed in $\mathrm{T} 1 \sim \mathrm{T} 12$, and (4) numeric rating scale (NRS) score $>6$. The exclusion criteria were as follows: (1) severe dysfunction of the heart, lung, liver, and kidney, (2) acute systemic infection or serious immune disorders, (3) infection of the skin overlying the vertebra of the affected dermatome, (4) coagulation abnormalities, (5) allergy to the drugs used in this study, and (6) psychiatric diseases. Eligible patients were randomly assigned to one of the two groups (the MB group or the control group) using a computer-generated random number list.

\section{Procedure}

After the patients were admitted to the hospital, routine examinations, including blood tests for coagulation, blood glucose, liver function, and kidney function, were performed. All treatment procedures were performed in the operating room. Standard monitors, including electrocardiogram, noninvasive blood pressure, pulse oximetry, and heart rate, were applied. A blinded theater nurse who did not participate in the present study opened the envelopes and determined the group assignment. The patients were placed in the prone position, and a thin pillow was placed under the patient's anterior lower thorax. The painful area on the body surface was labeled. Following standard skin asepsis, a 
sterile surgical towel was placed on the patient. Precise paravertebral catheter placement was performed using an S-Nerve ultrasound machine (Sonosite, Bothell, WA, USA) with a linear $5-10 \mathrm{MHz}$ ultrasound transducer at the thoracic level, using an in-plane approach. Prior to needle insertion, the skin was infiltrated with $3 \mathrm{ml}$ of $1 \%$ lidocaine. The ultrasound transducer was positioned parallel to the rib along with the targeted intercostal space. By moving the transducer position, we identified the wedgeshaped thoracic paravertebral space, which is a hypoechoic area containing the costotransverse ligament, parietal pleura, and transverse process on an ultrasound image. An 18-gauge Tuohy needle (Tuoren, Xinxiang, Henan, China) was inserted into the paravertebral space, approximately $5 \mathrm{ml}$ of normal saline was injected through the needle to dilate the paravertebral space, and a concomitant ventral shift of the pleura was confirmed. Subsequently, a 20-gauge epidural catheter (Tuoren, Xinxiang, Henan, China) was threaded through the needle into the paravertebral space $3-4 \mathrm{~cm}$ beyond the needle tip under real-time ultrasound surveillance. An aspiration test was performed to ensure that there was no intravascular or intrapleural puncture. The Tuohy needle was withdrawn, and the catheter was fixed to the skin. Finally, the catheter was connected to an electronic infusion pump and infused at a rate of $5 \mathrm{ml} / \mathrm{h}$. Patients in the $\mathrm{MB}$ group received a medication cocktail of $60 \mathrm{mg}$ methylene blue $6 \mathrm{ml}+1.5 \mathrm{~g}$ lidocaine hydrochloride $75 \mathrm{ml}+0.9 \% \mathrm{NaCl} 219 \mathrm{ml}$. Patients in the control group received a medication cocktail of $1.5 \mathrm{~g}$ lidocaine hydrochloride $75 \mathrm{ml}+0.9 \%$ $\mathrm{NaCl} 225 \mathrm{ml}$. The inserted catheter was maintained in position for 2.5 days.

\section{Outcome Measurement}

Preoperative data, including gender, age, weight, height, body mass index (BMI), duration of PHN, preoperative NRS, preoperative ISI, preoperative PHQ-9 score, and dosage of rescue medication, were recorded. Follow-up assessments were performed 1 week, 1 month, 3 months, and 6 months after the operation
(Fig. 1). Patients were evaluated at follow-up visits by a pain physician who did not participate in the surgery.

\section{Primary Outcome}

Pain intensity was assessed using the NRS. NRS scores were recorded in the two groups and ranged from 0 (no pain) to 10 (worst pain imaginable).

\section{Secondary Outcome}

\section{Depression Assessment}

The Patient Health Questionnaire-9 (PHQ-9) is a reliable and valid instrument for making diagnoses and assessing the severity of depressive disorders, and is scored as follows: $5-10$ points indicates mild depression, 10-15 points indicates moderate depression, 15-20 points indicates moderately severe depression, and $>20$ points indicates severe depression.

\section{Sleep Quality}

The Insomnia Severity Index (ISI) questionnaire was used to evaluate the severity of insomnia. The ISI is a validated 7-item self-report questionnaire that assesses insomnia severity over the past 2 weeks. A sum score was calculated (range 0-28), with lower scores indicating fewer insomnia symptoms.

\section{Effective Treatment Rate}

The treatment effect was assessed 6 months after the surgery according to the following criteria: "remarkable effect (RE)"-the symptoms and physical signs of the disease disappeared, with good quality of life restored; "valid effect (VE)" the symptoms and physical signs of the disease were relieved, with quality of life improved; "invalid effect (IE)"-no improvement in the symptoms, signs, or quality of life. Total effective rate $(\%)=[(\mathrm{RE}+\mathrm{VE}) / n] \times 100 \%$.

\section{Quality of Life}

Patient quality of life was accessed by the 36-Item Short-Form Health Survey (SF-36), which included the following domains: 


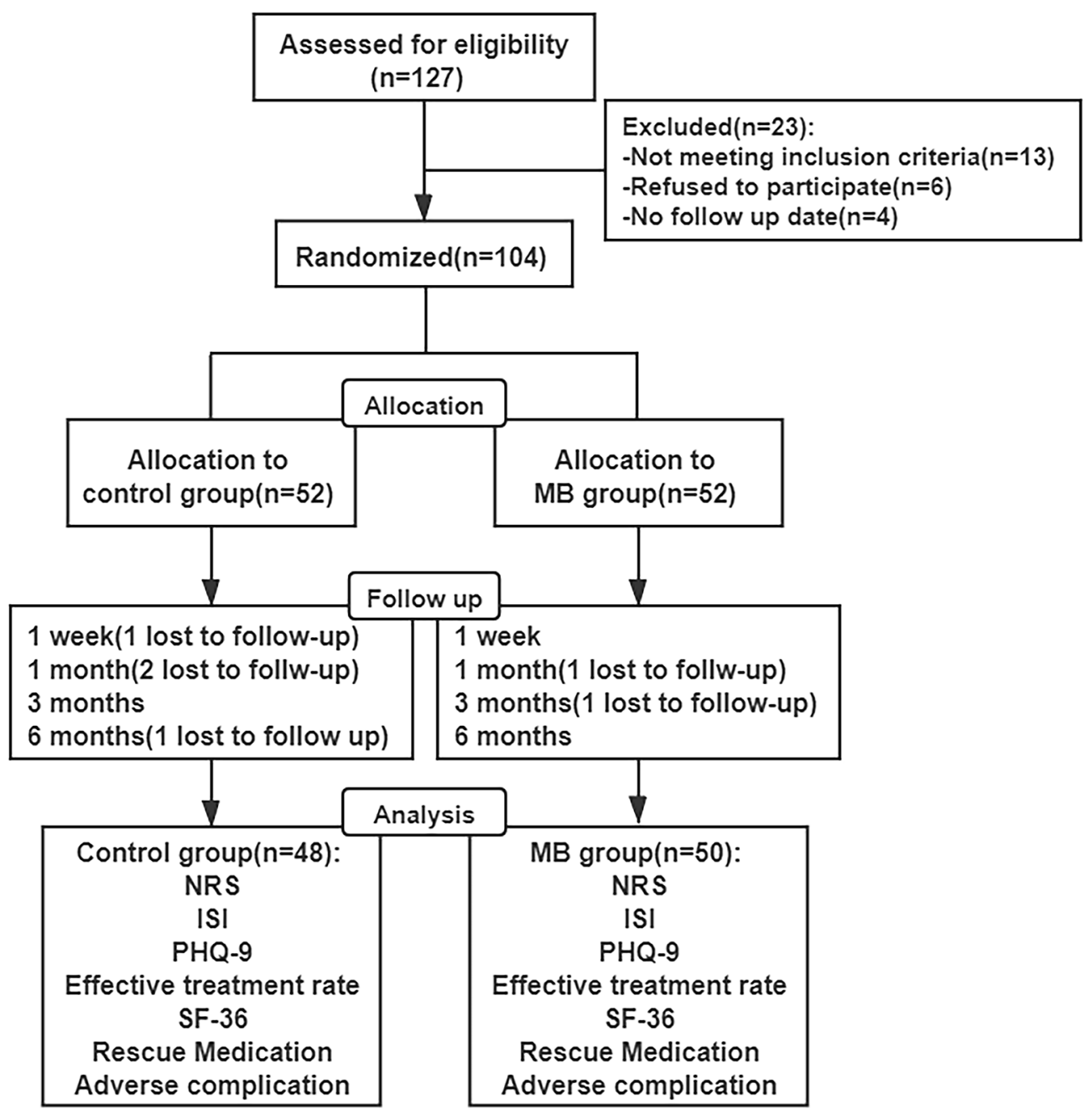

Fig. 1 Study flow diagram

physical function, role-physical, bodily pain, general health, vitality, social function, roleemotional, and mental health.

\section{Dosage of Rescue Medication}

Tramadol 50-100 mg twice a day and pregabalin $75-150 \mathrm{mg}$ twice a day orally were administered as rescue agents if the pain was not controlled sufficiently.

\section{Adverse Complications}

Possible complications, such as vascular puncture, hypotension, bradycardia, pleural puncture, pneumothorax, vertebral nerve puncture, catheter breakage, and lidocaine or methylene blue poisoning, were evaluated throughout the observation period.

\section{Statistical Analysis}

\section{Sample Size}

Because there was no reference for the effectiveness of continuous thoracic paravertebral infusion with $\mathrm{MB}$ in PHN patients, a preliminary trial was conducted before starting the formal research, as suggested and approved by the Institutional Review Board of Shanghai East Hospital. The preliminary trial indicated that the effective rate of treatment was $65 \%(13 / 20)$ in the control group and 90\% (18/20) in the MB group. Therefore, the sample size calculation was based on a $65 \%$ effective rate in the control group and a $90 \%$ effective rate in the MB group. Assuming a two-sided $\alpha=0.05$ and a statistical power of 0.8 , the sample size was calculated to 
be 40 for each group. Considering a 10\% loss to follow-up, the sample size was at least 44 in each group.

\section{Data Analysis}

Numerical variables are presented as the mean \pm standard deviation (SD), and categorical variables are reported as numbers or percentages. The Kolmogorov-Smirnov test was used to assess the normality of measurement data. Normally distributed data were analyzed using the independent $t$-test and repeated measures analysis of variance (ANOVA), and nonnormally distributed data were compared using the Mann-Whitney $U$ test. Differences between count data were calculated using the chi-square test or Fisher's exact test. A $p$ value $<0.05$ was considered statistically significant. Statistical analysis was performed using SPSS software (version 22; SPSS Inc., Chicago, IL, USA).

\section{RESULTS}

\section{Preoperative Patient Characteristics}

The demographic characteristics of the patients, including gender, age, height, weight, BMI, disease duration, pain intensity, and ISI and
PHQ-9 scores, were recorded before surgery, and no significant differences were found in these parameters between the two groups $(p>0.05$, Table 1).

\section{NRS Scores}

Compared with the preoperative baseline scores, the NRS scores at different time points were significantly decreased in both groups. The NRS scores were the lowest in the control group at 1 week and then gradually increased, but they were still lower than the preoperative baseline. Similarly, the NRS scores significantly declined at each time point after treatment in the MB group and were the lowest at 6 months. Compared with the control group, the MB group showed significantly lower NRS scores at each postoperative observation time point $(p<0.05$, Fig. 2).

\section{ISI Scores}

There was no significant difference in baseline ISI scores before the operation between the two groups. The ISI scores of the patients in the control group and MB group decreased significantly at each time point compared with the preoperative baseline. The ISI scores of the

Table 1 General preoperative characteristics of the patients (mean \pm SD)

\begin{tabular}{llll}
\hline Variable & Control group $(\boldsymbol{n}=\mathbf{4 8})$ & MB group $(\boldsymbol{n}=\mathbf{5 0})$ & $\boldsymbol{p}$-value \\
\hline Gender & & & 0.689 \\
Female & 28 & 27 & \\
Male & 20 & 23 & \\
Age & $62.21 \pm 7.15$ & $61.22 \pm 11.37$ & 0.632 \\
Height (cm) & $167.28 \pm 12.32$ & $168.93 \pm 12.17$ & 0.355 \\
Weight (kg) & $57.65 \pm 9.28$ & $59.11 \pm 12.38$ & 0.474 \\
BMI & $20.81 \pm 2.95$ & $21.02 \pm 3.18$ & 0.298 \\
PHN duration (months) & $3.89 \pm 2.02$ & $3.96 \pm 1.78$ & 0.524 \\
Preoperative NRS & $7.21 \pm 1.03$ & $7.40 \pm 0.97$ & 0.661 \\
Preoperative ISI & $21.31 \pm 4.27$ & $22.65 \pm 4.06$ & 0.494 \\
Preoperative PHQ-9 & $18.04 \pm 3.09$ & $18.92 \pm 3.03$ & 0.520 \\
\hline
\end{tabular}




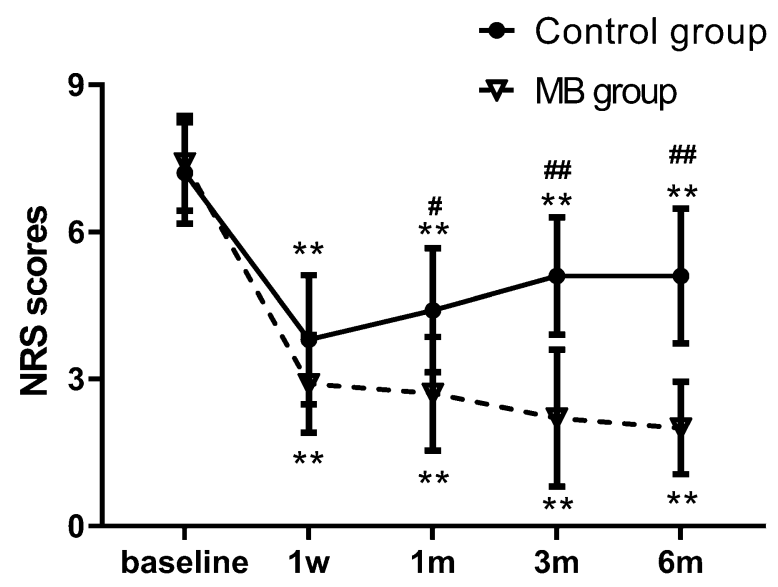

Fig. 2 Comparison of NRS scores in the two groups. The data are expressed as the mean $\pm \mathrm{SD} .{ }^{* *} p<0.01$ versus the preoperative value within the same group. ${ }^{\#} p<0.05$; $\# p<0.01$ versus the control group

control group were higher than those of the $\mathrm{MB}$ group at each time point ( $p<0.05$, Table 2$)$.

\section{PHQ-9 Scores}

There was no significant difference in preoperative baseline PHQ-9 scores between the two groups. The PHQ-9 scores of the patients in the two groups decreased significantly at each time point compared with the preoperative baseline. The PHQ-9 scores of the control group were higher than those of the MB group at each time point $(p<0.05$, Table 3$)$.

\section{Total Effective Rate}

The total effective rate was $64.58 \%$ in the control group 6 months after the surgery. In the MB group, the total effective rate was $88.00 \%$ 6 months after the surgery $(p<0.05$, Table 4$)$.

\section{SF-36 Assessment}

There was no significant difference between the groups in the preoperative baseline SF-36 score, but the SF-36 scores in domains including general health, body pain, physical function, rolephysical, vitality, mental health, role-emotional, and social function evidently improved in the two groups at each observation time point compared to the preoperative baseline $(p<0.05$; Fig. 3$)$; the difference between the

Table 2 Comparison of ISI scores in the two groups (mean \pm SD)

\begin{tabular}{lllr}
\hline ISI score & Control group $(\boldsymbol{n}=\mathbf{4 8})$ & MB group $(\boldsymbol{n}=\mathbf{5 0})$ & $\boldsymbol{p}$-value \\
\hline Preoperative & $21.31 \pm 4.27$ & $22.65 \pm 4.06$ & 0.494 \\
1 week postoperative & $15.40 \pm 2.53^{\mathrm{a}}$ & $13.10 \pm 2.13^{\mathrm{a}}$ & 0.040 \\
1 month postoperative & $16.00 \pm 2.63^{\mathrm{a}}$ & $11.33 \pm 2.06^{\mathrm{a}}$ & $<0.01$ \\
3 months postoperative & $16.13 \pm 3.48^{\mathrm{a}}$ & $9.93 \pm 1.97^{\mathrm{a}}$ & $<0.01$ \\
6 months postoperative & $15.82 \pm 3.62^{\mathrm{a}}$ & $9.26 \pm 1.93^{\mathrm{a}}$ & $<0.01$ \\
\hline
\end{tabular}

${ }^{a} p<0.05$ versus the preoperative value within the same group

Table 3 Comparison of PHQ-9 scores in the two groups (mean \pm SD)

\begin{tabular}{lllr}
\hline PHQ-9 score & Control group $(\boldsymbol{n}=\mathbf{4 8})$ & MB group $(\boldsymbol{n}=\mathbf{5 0})$ & $\boldsymbol{p}$-value \\
\hline Preoperative & $18.04 \pm 3.09$ & $18.92 \pm 3.03$ & 0.520 \\
1 week postoperative & $11.71 \pm 2.06^{\mathrm{a}}$ & $10.70 \pm 2.58^{\mathrm{a}}$ & 0.351 \\
1 month postoperative & $13.37 \pm 2.50^{\mathrm{a}}$ & $6.63 \pm 2.012^{\mathrm{a}}$ & $<0.01$ \\
3 months postoperative & $12.20 \pm 4.21^{\mathrm{a}}$ & $5.64 \pm 1.85^{\mathrm{a}}$ & $<0.01$ \\
6 months postoperative & $12.66 \pm 3.57^{\mathrm{a}}$ & $4.05 \pm 1.76^{\mathrm{a}}$ & $<0.01$ \\
\hline
\end{tabular}

${ }^{\mathrm{a}} p<0.05$ versus the preoperative value within the same group 
Table 4 Comparison of the total effective rates in the two groups

\begin{tabular}{lllll}
\hline Group & Remarkable & Valid & Invalid & Total effective rate \\
\hline Control group $(n=48)$ & 10 & 21 & 17 & $64.58 \%$ \\
MB group $(n=50)$ & 21 & 23 & 6 & $88.00 \%^{\mathrm{a}}$ \\
\hline
\end{tabular}

${ }^{a} p<0.05$ versus the control group

control group and $\mathrm{MB}$ group was statistically significant after 1 week, and a statistically significant difference was maintained for 6 months $(p<0.05$; Fig. 3).

\section{Dosage of Rescue Medication}

The rescue drug (pregabalin and tramadol) daily dosages were both significantly reduced at each time point after the operation compared to the preoperative baseline. The daily dosages of pregabalin and tramadol were lower in the $\mathrm{MB}$ group than in the control group at each time point after surgery ( $p<0.05$; Fig. 4 ).

\section{Adverse Complications}

No adverse complications, such as vascular puncture, hypotension, bradycardia, pleural puncture, pneumothorax, vertebral nerve puncture, catheter breakage, and lidocaine or methylene blue poisoning, occurred in either group during the follow-up period. No patients withdrew from the treatment because of adverse complications.

\section{DISCUSSION}

Postherpetic neuralgia (PHN) is the most frequent chronic complication of herpes zoster (HZ) and is characterized by persistent skin burning or knife-like neuralgia in the affected area. PHN is a chronic neuropathic pain syndrome that can persist for more than 3 months after the initial onset of a $\mathrm{HZ}$ rash. PHN seriously affects patient quality of life and can cause physical disability, anxiety, depression, insomnia, and social isolation; it also presents serious economic burdens and public health problems for society [1, 24, 25]. Although there are various treatments for PHN, they are not fully effective at relieving PHN.

Methylene blue (MB) is an inhibitor of nitric oxide synthase (NOS) and soluble guanylate cyclase (sGC), which can be used in the treatment of methemoglobinemia [18], cyanide poisoning [26], carbon monoxide poisoning [27], pruritus ani [28], vasoplegia [28], malaria [29], osteoarthritis [19] and ifosfamide-induced encephalopathy [30]. In addition, Miclescu et al. showed that intravenous infusion of $\mathrm{MB}$ effectively decreased pain levels in patients with chronic therapy-resistant neuropathic pain [31]. Several studies have demonstrated that injection of MB into a painful disc is a safe, effective, and minimally invasive method for the treatment of intractable and incapacitating discogenic low back pain $[32,33]$. Recently, Carlos et al. found that $\mathrm{MB}$ oral rinse significantly reduced refractory neuropathic pain from oral mucositis related to cancer treatment [34]. The abovementioned evidence indicates that $\mathrm{MB}$ is a safe and useful analgesic for various painful conditions. In the present study, we comprehensively assessed the analgesic effects of continuous $\mathrm{MB}$ thoracic paravertebral infusion in patients with moderate-to-severe PHN. We found that the NRS scores of PHN patients in both of the studied groups were significantly lower over the 6-month follow-up period than at baseline. Furthermore, NRS scores were significantly lower in the MB group than in the control group from 1 to 6 months after the surgery. We also found that continuous thoracic paravertebral infusion with $\mathrm{MB}$ can effectively reduce the daily doses of oral rescue drugs (pregabalin and tramadol).

Several possible mechanisms may be involved in the relief of neuropathic pain by MB. First, activation of $N$-methyl-D-aspartate (NMDA) receptors in the spinal cord dorsal horn can increase calcium conductance and activate 

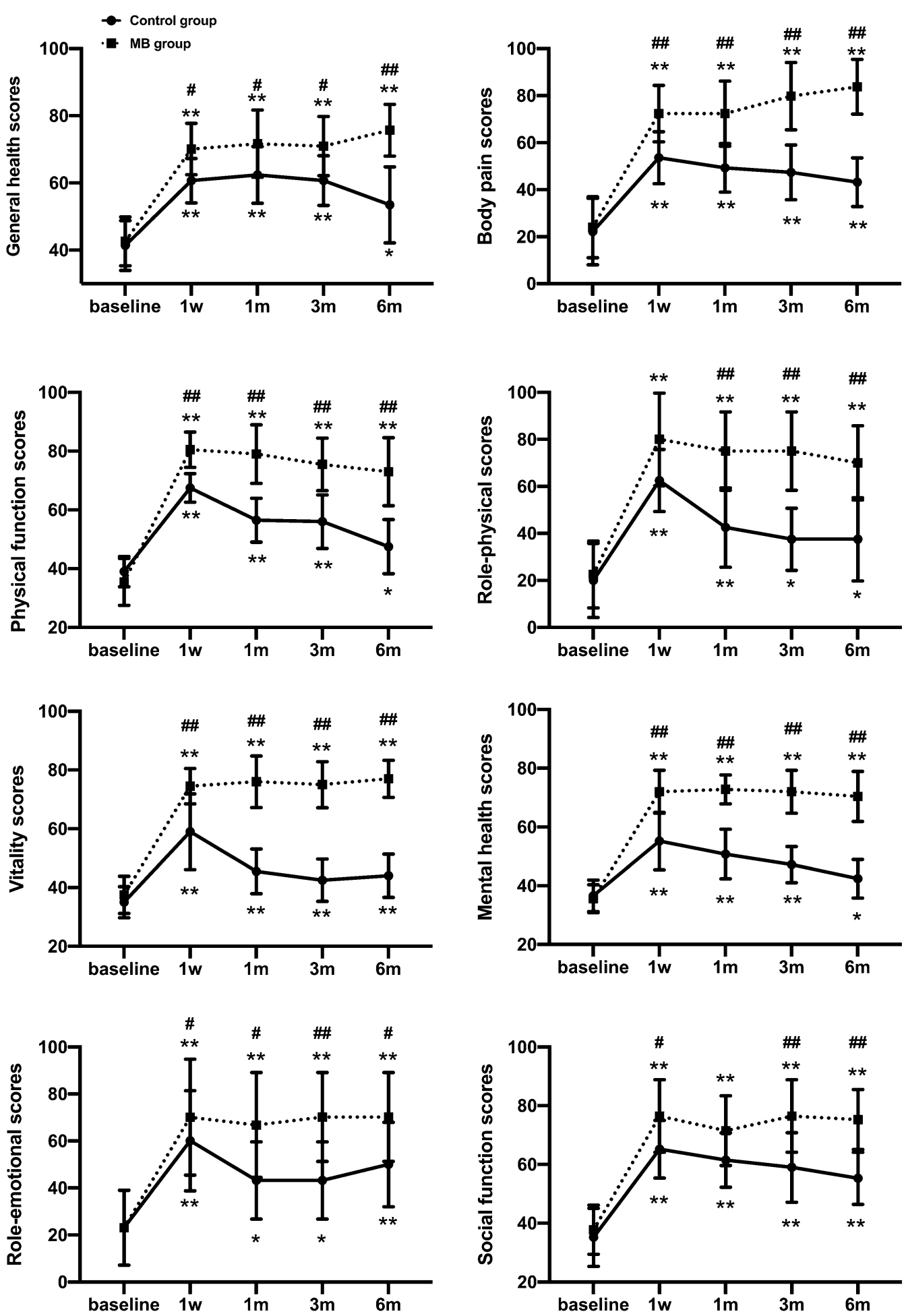

Fig. 3 Comparison of quality of life scores (SF-36) in the two groups. The data are expressed as the mean \pm SD. ${ }^{*} p<0.05$; ${ }^{* *} p<0.01$ versus the preoperative value within the same group. ${ }^{\#} p<0.05 ;{ }^{\#} p<0.01$ versus the control group 


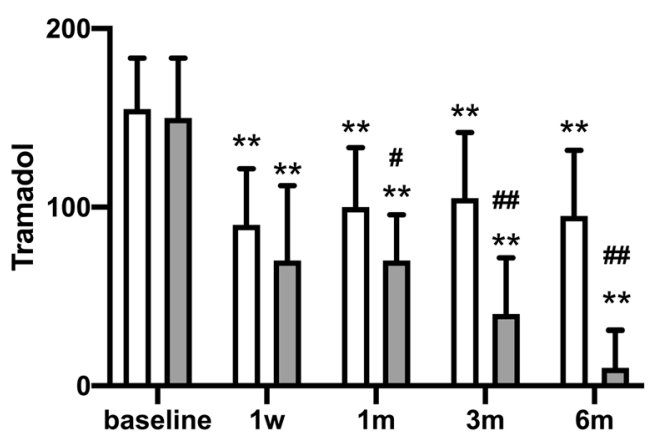

Fig. 4 Comparison of the daily dosages of pregabalin and tramadol in the two groups. The data are expressed as the mean $\pm \mathrm{SD} .{ }^{* *} p<0.01$ versus the preoperative value

NOS, ultimately modulating the NO/cyclic guanosine monophosphate (cGMP)-dependent pathway, which enables low-threshold mechanosensitive $A \beta$ - and $A \delta$-afferent fibers to activate central pain pathways. $\mathrm{MB}$, as an inhibitor of NOS and sGC, can effectively block these pathways and shows antinociceptive effects [35-37]. Second, neuroinflammation contributes significantly to the development and maintenance of neuropathic pain, which results from the activation of glial cells, including microglia and astrocytes. Activated microglia and astrocytes release proinflammatory cytokines, such as tumor necrosis factor (TNF)- $\alpha$, interleukin (IL)-1 $\beta$, and chemokines, to activate and sensitize spinal cord nociceptive neurons [38-40], while MB has been reported to have anti-inflammatory properties in a series of disease models [41-43]. Recently, Ping et al. found that $\mathrm{MB}$ thoracic paravertebral single injection effectively inhibited the levels of plasma IL- 6 , TNF- $\alpha$, and cortisol in PHN patients [23], indicating that the analgesic effect of $\mathrm{MB}$ may be related to its anti-inflammatory properties. Furthermore, some studies found that MB exerted its beneficial effect by attenuating mitochondrial dysfunction-induced oxidative stress [44-46]. However, the therapeutic mechanism of $\mathrm{MB}$ for PHN needs to be further investigated.

PHN may persist for years and is difficult to treat. Current guidelines suggest that treatment with alpha-2 delta ligands, tricyclic antidepressants, opioid analgesics, lidocaine patch, or

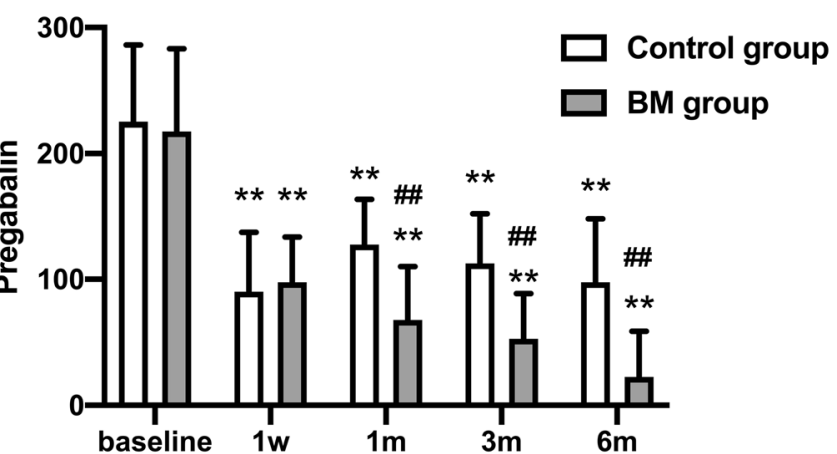

within the same group. ${ }^{\#} p<0.05 ;{ }^{\# \#} p<0.01$ versus the control group

capsaicin cream should be initiated. In addition, nerve block, intrathecal glucocorticoid injection, local botulinum toxin A injection, pulsed radiofrequency, spinal cord stimulation, dorsal root ganglion stimulation, and physical therapy are routine treatments for PHN $[8,9,46,47]$. Thoracic and cervical dermatome involvement is reportedly most common in patients with HZ [48, 49]. TPVB can produce ipsilateral somatosensory and sympathetic nerve blockade in the thoracic dermatome; therefore, it is an ideal and effective therapy for the treatment of acute thoracic $\mathrm{HZ}$ pain and PHN. Several studies have proven that TPVB can remarkably lower the pain intensity in thoracic acute $\mathrm{HZ}$ pain, and it also effectively decreases the incidence of PHN [50-52]. More recently, Ping et al. indicated that a single thoracic paravertebral injection with $\mathrm{MB}$ can effectively lower the pain levels of PHN patients within 1 month after treatment [23]; however, this study lacked a control group, and the long-term analgesic efficacy of $\mathrm{MB}$ for the treatment of PHN needs further investigation. In the present study, we found that continuous thoracic paravertebral injection with $\mathrm{MB}$ evidently decreased the NRS scores in PHN patients, and that this therapeutic effect can last for at least 6 months, indicating that continuous paravertebral injection with $\mathrm{MB}$ can provide a longer postoperative analgesic effect in PHN patients.

Chronic pain can dramatically increase the risk of developing insomnia, anxiety, and depression, while it has a negative effect on the 
patient's quality of life [53, 54]. Several studies have proven that PHN patients have abnormal activities in brain regions related to anxiety and depression, such as the limbic system and frontal lobe $[55,56]$. Therefore, we investigated the effect of continuous MB infusion on insomnia, depression, and quality of life in PHN patients. Our results showed that the PHN patients in both groups obtained significantly decreased insomnia (ISI) scores and depression (PHQ-9) scores and experienced improved quality of life. In addition, patients in the MB group had lower insomnia scores and depression scores and better quality of life than those in the control group. Currently, ultrasoundguided nerve blocks are widely performed for regional anesthesia, postoperative analgesia, and chronic pain management. Traditionally, TPVB mainly uses either the "landmark technique" or "loss of resistance" methods to locate the thoracic paravertebral space; this is a difficult technique among ultrasound-guided nerve blocks because the paravertebral space is not visualized in real time [57-59]. The failure rate of traditional TPVB is $7-10 \%$, and its application may lead to pleural, vascular, and nerve damage that further causes pneumothorax, hematoma, and other adverse complications [60]. In contrast to traditional TPVB, ultrasound guidance has significantly improved the safety and efficacy of TPVB, and ultrasound-guided TPVB (USG-TPVB) can provide real-time and accurate visualization of the needle tip and precise placement of catheters to allow continuous anesthesia and pain management. In this study, the catheter was successfully inserted into the thoracic paravertebral space and fixed to the skin of PHN patients, and no adverse effects or complications were reported in either group.

There are also several limitations to our study. First, the patients were recruited from a single center and the sample size was small. Second, the patients were only followed for 6 months after treatment. Third, double blinding was not used in this research.

\section{CONCLUSIONS}

In conclusion, ultrasound-guided continuous thoracic paravertebral infusion with $\mathrm{MB}$ is a novel, safe, and effective therapy for PHN. Continuous infusion with MB can significantly reduce pain intensity, improve pain-related depression, increase quality of life, and decrease the amount of rescue medicine needed while incurring no serious adverse side effects.

\section{ACKNOWLEDGEMENTS}

We thank the participants of the study. We thank Zhenzhen Lu, Yidan Jiang for helpful discussions and statistical review.

Funding. This work was supported by the National Natural Science Foundation of China (Nos. 82000085, 81971814, 81871601), the Young Elite Scientist Sponsorship Program by CAST (2018QNRC001), the Development Fund for Anesthesiology by Shanghai Pulmonary Hospital, the Basic Research Program for Young Elite Scientist by Shanghai Association for the Study of Pain (2018SASP01), the Research Program for Young Scientist by Shanghai Society of Anesthesiology (2019SSA), Shanghai Pujiang Talent Program (2020PJD050), Discipline Leader Training Program of Health and Family Planning Commission of Pudong New Area (PWRd2020-06). The journal's Rapid Service Fee funded by the authors.

Authorship. All named authors meet the International Committee of Medical Journal Editors (ICMJE) criteria for authorship for this article, take responsibility for the integrity of the work as a whole, and have given their approval for this version to be published.

Authorship Contributions. Mingxia Wang, Jinyuan Zhang, Li Zheng contribute to study design, analysis, and manuscript drafting. Hongwei Fang, Yiguo Zhang, Huimin Deng contribute to data analysis and manuscript editing. Mansi Wang, Xiuqin Yu, Qingxiang Meng, Yuanli Chen contribute to patient 
follow-up, data collection. Lijun Liao, Xin Lv contribute to study concept, design. Hao Yang, Xiangrui Wang contribute to study concept, design, analysis, and manuscript editing.

Disclosures. Mingxia Wang, Jinyuan Zhang, Li Zheng, Hongwei Fang, Yiguo Zhang, Huimin Deng, Mansi Wang, Xiuqin Yu, Qingxiang Meng, Yuanli Chen, Lijun Liao, Xin Lv, Hao Yang, and Xiangrui Wang have nothing to disclose.

Compliance with Ethics Guidelines. This study was approved by the Institutional Review Board and Ethics Committee of Shanghai East Hospital (2019, No.006). The present clinical research was conducted in accordance with the Declaration of Helsinki. Written informed consent was obtained from all patients before inclusion.

Data Availability. The datasets generated during and/or analyzed during the current study are available from the corresponding author on reasonable request.

Open Access. This article is licensed under a Creative Commons Attribution-NonCommercial 4.0 International License, which permits any non-commercial use, sharing, adaptation, distribution and reproduction in any medium or format, as long as you give appropriate credit to the original author(s) and the source, provide a link to the Creative Commons licence, and indicate if changes were made. The images or other third party material in this article are included in the article's Creative Commons licence, unless indicated otherwise in a credit line to the material. If material is not included in the article's Creative Commons licence and your intended use is not permitted by statutory regulation or exceeds the permitted use, you will need to obtain permission directly from the copyright holder. To view a copy of this licence, visit http://creativecommons.org/licenses/bync/4.0/.

\section{REFERENCES}

1. Johnson RW, Rice AS. Clinical practice. Postherpetic neuralgia. N Engl J Med. 2014;371:1526-33.

2. Ngo AL, Urits I, Yilmaz M, Fortier L, Anya A, Oh JH, et al. Postherpetic neuralgia: current evidence on the topical film-forming spray with bupivacaine hydrochloride and a review of available treatment strategies. Adv Ther. 2020;37:2003-16.

3. Insinga RP, Itzler RF, Pellissier JM, Saddier P, Nikas AA. The incidence of herpes zoster in a United States administrative database. J Gen Intern Med. 2005;20:748-53.

4. Massengill JS, Kittredge JL. Practical considerations in the pharmacological treatment of postherpetic neuralgia for the primary care provider. J Pain Res. 2014;7:125-32.

5. Yang F, Yu S, Fan B, Liu Y, Chen YX, Kudel I, et al. The epidemiology of herpes zoster and postherpetic neuralgia in China: results from a cross-sectional study. Pain Ther. 2019;8:249-59.

6. Kawai K, Rampakakis E, Tsai TF, Cheong HJ, Dhitavat J, Covarrubias AO, et al. Predictors of postherpetic neuralgia in patients with herpes zoster: a pooled analysis of prospective cohort studies from North and Latin America and Asia. Int J Infect Dis. 2015;34:126-31.

7. Johnson RW, Bouhassira D, Kassianos G, Leplège A, Schmader KE, Weinke T. The impact of herpes zoster and post-herpetic neuralgia on quality-oflife. BMC Med. 2010;8:37.

8. Mallick-Searle T, Snodgrass B, Brant JM. Postherpetic neuralgia: epidemiology, pathophysiology, and pain management pharmacology. J Multidiscip Healthc. 2016;9:447-54.

9. Lin CS, Lin YC, Lao HC, Chen CC. Interventional treatments for postherpetic neuralgia: a systematic review. Pain Physician. 2019;22:209-28.

10. Wang Y, Li W, Peng W, Zhou J, Liu Z. Acupuncture for postherpetic neuralgia: systematic review and meta-analysis. Medicine. 2018;97:e11986.

11. Makharita MY, Amr YM, El-Bayoumy Y. Effect of early stellate ganglion blockade for facial pain from acute herpes zoster and incidence of postherpetic neuralgia. Pain Physician. 2012;15:467-74.

12. Ueshima H. Thoracoabdominal nerve block through perichondral approach for pain management of thoracic post-herpetic neuralgia. J Clin Anesth. 2019;55:110. 
13. Dong X, Liu Y, Yang Q, Liu Z, Zhang Z. Comparison of therapeutic effects of continuous epidural nerve block combined with drugs on postherpetic neuralgia. Int J Neurosci. 2021;131:191-5.

14. Okajima H, Tanaka O, Ushio M, Higuchi Y, Nagai Y, Iijima $\mathrm{K}$, et al. Ultrasound-guided continuous thoracic paravertebral block provides comparable analgesia and fewer episodes of hypotension than continuous epidural block after lung surgery. J Anesth. 2015;29:373-8.

15. Jin L, Yao R, Heng L, Pang B, Sun FG, Shen Y, et al. Ultrasound-guided continuous thoracic paravertebral block alleviates postoperative delirium in elderly patients undergoing esophagectomy: a randomized controlled trial. Medicine. 2020;99: e19896.

16. Kim J, Lee HJ, Lee YJ, Lee CS, Yoo Y, Moon JY. Ultrasound-guided thoracic paravertebral block as a sympathetic blockade for upper extremity neuropathic pain: a prospective pilot study. J Pain Res. 2020;13:3395-403.

17. Womack J, Pearson JD, Walker IA, Stephens NM, Goodman BA. Safety, complications and clinical outcome after ultrasound-guided paravertebral catheter insertion for rib fracture analgesia: a singlecentre retrospective observational study. Anaesthesia. 2019;74:594-601.

18. Ginimuge PR, Jyothi SD. Methylene blue: revisited. J Anaesthesiol Clin Pharmacol. 2010;26:517-20.

19. Zheng J, Li Q. Methylene blue regulates inflammatory response in osteoarthritis by noncoding long chain RNA CILinc02. J Cell Biochem. 2019;120: 3331-8.

20. Genrikhs EE, Stelmashook EV, Voronkov DN, Novikova SV, Alexandrova OP, Fedorov AV, et al. The single intravenous administration of methylene blue after traumatic brain injury diminishes neurological deficit, blood-brain barrier disruption and decrease in the expression of S100 protein in rats. Brain Res. 2020;1740:146854.

21. Berrocal M, Corbacho I, Gutierrez-Merino C, Mata AM. Methylene blue activates the PMCA activity and cross-interacts with amyloid $\beta$-peptide, blocking A $\beta$-mediated PMCA inhibition. Neuropharmacology. 2018;139:163-72.

22. Jang DH, Donovan S, Nelson LS, Bania TC, Hoffman RS, Chu J. Efficacy of methylene blue in an experimental model of calcium channel blockerinduced shock. Ann Emerg Med. 2015;65:410-5.

23. Zhao P, Mei L, Wang W. Clinical study of ultrasound-guided methylene blue thoracic paravertebral nerve block for the treatment of postherpetic neuralgia. Turk Neurosurg. 2019;29: 811-5.

24. Saguil A, Kane S, Mercado M, Lauters R. Herpes zoster and postherpetic neuralgia: prevention and management. Am Fam Physician. 2017;96:656-63.

25. Sampathkumar P, Drage LA, Martin DP. Herpes zoster (shingles) and postherpetic neuralgia. Mayo Clin Proc. 2009;84:274-80.

26. Haouzi P, Gueguinou $M$, Sonobe $T$, JudenhercHaouzi A, Tubbs N, Trebak M, et al. Revisiting the physiological effects of methylene blue as a treatment of cyanide intoxication. Clin Toxicol (Phila). 2018;56:828-40.

27. Draize JH. Sodium tetrathionate and methylene blue in cyanide and carbon monoxide poisoning. Science (New York, NY). 1933;78:145.

28. Kim JH, Kim DH, Lee YP. Long-term follow-up of intradermal injection of methylene blue for intractable, idiopathic pruritus ani. Tech Coloproctol. 2019;23:143-9.

29. Rengelshausen J, Burhenne J, Fröhlich M, Tayrouz Y, Singh SK, Riedel KD, et al. Pharmacokinetic interaction of chloroquine and methylene blue combination against malaria. Eur J Clin Pharmacol. 2004;60:709-15.

30. Turner AR, Duong CD, Good DJ. Methylene blue for the treatment and prophylaxis of ifosfamide-induced encephalopathy. Clin Oncol. 2003;15:435-9.

31. Miclescu AA, Svahn M, Gordh TE. Evaluation of the protein biomarkers and the analgesic response to systemic methylene blue in patients with refractory neuropathic pain: a double-blind, controlled study. J Pain Res. 2015;8:387-97.

32. Zhang X, Hao J, Hu Z, Yang H. Clinical evaluation and magnetic resonance imaging assessment of intradiscal methylene blue injection for the treatment of discogenic low back pain. Pain Physician. 2016;19:E1189-95.

33. Kallewaard JW, Geurts JW, Kessels A, Willems P, van Santbrink H, van Kleef M. Efficacy, safety, and predictors of intradiscal methylene blue injection for discogenic low back pain: results of a multicenter prospective clinical series. Pain Pract. 2016;16:405-12.

34. Roldan CJ, Chung M, Feng L, Bruera E. Methylene blue for the treatment of intractable pain from oral mucositis related to cancer treatment: an uncontrolled cohort. J Natl Comprehens Cancer Netw JNCCN. 2021;2:1-7. 
35. Schmidtko A, Tegeder I, Geisslinger G. No NO, no pain? The role of nitric oxide and cGMP in spinal pain processing. Trends Neurosci. 2009;32:339-46.

36. Patil CS, Padi SV, Singh VP, Kulkarni SK. Sildenafil induces hyperalgesia via activation of the NOcGMP pathway in the rat neuropathic pain model. Inflammopharmacology. 2006;14:22-7.

37. Komatsu T, Sakurada S, Kohno K, Shiohira H, Katsuyama S, Sakurada C, et al. Spinal ERK activation via NO-cGMP pathway contributes to nociceptive behavior induced by morphine-3-glucuronide. Biochem Pharmacol. 2009;78:1026-34.

38. Myers RR, Campana WM, Shubayev VI. The role of neuroinflammation in neuropathic pain: mechanisms and therapeutic targets. Drug Discov Today. 2006;11:8-20.

39. Zhou D, Zhang S, Hu L, Gu YF, Cai Y, Wu D, et al. Inhibition of apoptosis signal-regulating kinase by paeoniflorin attenuates neuroinflammation and ameliorates neuropathic pain. J Neuroinflammation. $2019 ; 16: 83$.

40. Yang H, Wu L, Deng H, Chen Y, Zhou H, Liu M, et al. Anti-inflammatory protein TSG- 6 secreted by bone marrow mesenchymal stem cells attenuates neuropathic pain by inhibiting the TLR2/MyD88/ NF- $\kappa \mathrm{B}$ signaling pathway in spinal microglia. J Neuroinflammation. 2020;17:154.

41. Lin ZH, Wang SY, Chen LL, Zhuang JY, Ke QF, Xiao $\mathrm{DR}$, et al. Methylene blue mitigates acute neuroinflammation after spinal cord injury through inhibiting NLRP3 inflammasome activation in microglia. Front Cell Neurosci. 2017;11:391.

42. Chen C, Zhou F, Zeng L, Jiang Z, Hu Z. Methylene blue offers neuroprotection after intracerebral hemorrhage in rats through the PI3K/Akt/GSK3 $\beta$ signaling pathway. J Cell Physiol. 2019;234: 5304-18.

43. Xu H, Li J, Wang Z, Feng M, Shen Y, Cao S, et al. Methylene blue attenuates neuroinflammation after subarachnoid hemorrhage in rats through the Akt/GSK-3 $\beta /$ MEF2D signaling pathway. Brain Behav Immun. 2017;65:125-39.

44. Tucker D, Lu Y, Zhang Q. From mitochondrial function to neuroprotection-an emerging role for methylene blue. Mol Neurobiol. 2018;55:5137-53.

45. Yang L, Youngblood H, Wu C, Zhang Q. Mitochondria as a target for neuroprotection: role of methylene blue and photobiomodulation. Transl Neurodegen. 2020;9:19.

46. Deng Y, Wang R, Li S, Zhu X, Wang T, Wu J, et al. Methylene blue reduces incidence of early postoperative cognitive disorders in elderly patients undergoing major non-cardiac surgery: an openlabel randomized controlled clinical trial. J Clin Anesth. 2021;68:110108.

47. Piedade GS, Vesper J, Chatzikalfas A, Slotty PJ. Cervical and high-thoracic dorsal root ganglion stimulation in chronic neuropathic pain. Neuromodulation. 2019;22:951-5.

48. Le P, Rothberg M. Herpes zoster infection. BMJ. 2019;364:k5095.

49. Nayak MK, Kapadia JD, Desai CK, Desai MK, Shah BJ. An evaluation of efficacy and safety of commonly prescribed drugs and effect of these drugs on quality of sleep in patients suffering from zosterassociated pain. J Clin Pharmacol. 2018;58: 1406-17.

50. Makharita MY, Amr YM. Effect of repeated paravertebral injections with local anesthetics and steroids on prevention of post-herpetic neuralgia. Pain Physician. 2020;23:565-72.

51. Makharita MY, Amr YM, El-Bayoumy Y. Single paravertebral injection for acute thoracic herpes zoster: a randomized controlled trial. Pain Pract. 2015;15:229-35.

52. Zhao P, Mei L. A clinical study of paraspinal nerve block on treatment of herpes zoster under ultrasonic guidance. Neurochirurgie. 2019;65:382-6.

53. Cherif F, Zouari HG, Cherif W, Hadded M, Cheour M, Damak R. Depression prevalence in neuropathic pain and its impact on the quality of life. Pain Res Manag. 2020;2020:7408508.

54. Fang H, Zhang J, Yang Y, Ye L, Wang X. Clinical effect and safety of pulsed radiofrequency treatment for pudendal neuralgia: a prospective, randomized controlled clinical trial. J Pain Res. 2018;11:2367-74.

55. Cao S, Song G, Zhang Y, Xie P, Tu Y, Li Y, et al. Abnormal local brain activity beyond the pain matrix in postherpetic neuralgia patients: a restingstate functional MRI study. Pain Physician. 2017;20:E303-14.

56. Cao S, Li Y, Deng W, Qin B, Zhang Y, Xie P, et al. Local brain activity differences between herpes zoster and postherpetic neuralgia patients: a resting-state functional MRI study. Pain Physician. 2017;20:E687-99.

57. Uppal V, Sondekoppam RV, Sodhi P, Johnston D, Ganapathy S. Single-injection versus multiple-injection technique of ultrasound-guided paravertebral blocks: a randomized controlled study 
comparing dermatomal spread. Reg Anesth Pain Med. 2017;42:575-81.

58. Karmakar MK. Thoracic paravertebral block. Anesthesiology. 2001;95:771-80.

59. Okitsu K, Maeda A, Iritakenishi T, Fujino Y. The feasibility of pressure measurement during an ultrasound-guided thoracic paravertebral block. Eur J Anaesthesiol. 2018;35:806-7.

60. El-Morsy GZ, El-Deeb A, El-Desouky T, Elsharkawy AA, Elgamal MA. Can thoracic paravertebral block replace thoracic epidural block in pediatric cardiac surgery? A randomized blinded study. Ann Card Anaesth. 2012;15:259-63. 